\title{
Signal Model-Based Fault Detection and Diagnosis for Induction Motors Using Features of Vibration Signal in Two- Dimension Domain
}

\author{
Van Tuan Do1,* - Ui-Pil Chong2 \\ 1 VTT Technical Research Centre of Finland, Finland \\ ${ }^{2}$ University of Ulsan, Department of Computer Engineering and Information Technology, South Korea
}

In this paper, we propose an approach for vibration signal-based fault detection and diagnosis system applying for induction motors. The approach consists of two consecutive processes: fault detection process and fault diagnosis process. In the fault detection process, significant features from vibration signals are extracted through the scale invariant feature transform (SIFT) algorithm to generate the faulty symptoms. Consequently, the pattern classification technique using the faulty symptoms is applied to the fault diagnosis process. Hence, instead of analyzing the vibration signal to determine the induction motor faults, the vibration signal can be classified to the corresponding faulty category, which presents the induction motor fault. We also provide a framework for the pattern classification technique that is applicable to SIFT patterns. Moreover, a comparison with two other approaches in our previous work is also carried out. The testing results show that our proposed approach provides significantly high fault classification accuracy and a better performance than previous approaches.

(C)2011 Journal of Mechanical Engineering. All rights reserved.

Keywords: fault detection and diagnosis, SIFT, feature vector, texton dictionary, two-dimension domain, classification accuracy

\section{INTRODUCTION}

Systems for detection and diagnosis of malfunctioning machines play an important role in industrial fields. They are critical in the manufacturing industry, since a bad manufacturing machine may produce many defective products dangerous to consumers. An investigation for the earliest possible detection for a machine before it becomes faulty is therefore compulsory.

The approaches for fault detection and diagnosis consist of two processes: fault detection process and fault diagnosis process as shown in Fig. 1. The fault detection process analyzes the measured signals such as vibration, noise, acoustic sound, pressure or bases on the analytical parameters to generate the faulty symptoms, which can be analytical symptoms or heuristic symptoms [1] to [2]. The faulty symptoms are the input of the fault diagnosis process that determines the size, type and location of the system fault [3].

Depending on a specific application, simple or complex techniques for fault detection process and fault diagnosis process can be applied.

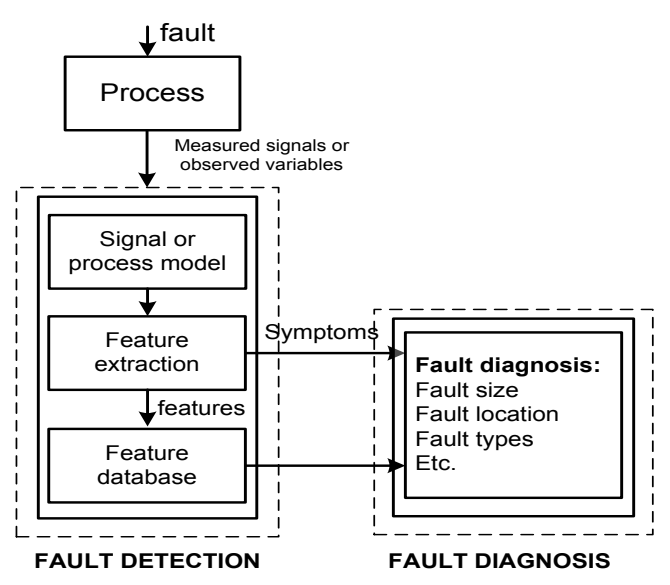

Fig. 1. General structure of model-based fault detection and diagnosis

For example, limit checking techniques are widely used for simple fault detection systems. However, they have two disadvantages: it is impossible to predict the fault in advance since the fault has already occurred when detected and the methods do not provide the type, size and location of faults, which can be provided by applying

*Corr. Author's Address: VTT Technical Research Centre of Finland, FI-02044, Espoo, Finland, dtuan@ualberta.ca 
model-based fault detection technique such as approaches in [4] to [6].

The Induction motor is a three phase AC motor and is the most widely used machine. Its characteristic features are: simple and rugged construction, low cost and minimum maintenance, high reliability and sufficiently high efficiency, it needs no extra starting motor and need not be synchronized. During the operation; however, there are several types of faults frequently happening such as: bearing faults, gear faults, rotor bar eccentricity and stator winding failures and misalignment. The fault detection and diagnosis methods for induction motors are wide such as current spectrum analysis, vibration analysis and acoustic analysis for different types of motor fault identification [7] to [10]. Since previous research showed that more than 40 percent of faults in induction motors are related to bearing faults [11], a number of research works have been done with bearing fault detection using wavelet technique for vibration data [12] to [13]. Gear faults are also common in the induction motor. In [14], the authors used an adaptive method for vibration to detect the gear tooth faults. Neural networks and classification techniques also widely used in fault diagnosis [15] to [17].

In this paper, a new approach for fault detection and diagnosis system for induction motors in which the fault detection process is based on vibration signals and the fault diagnosis process is based on the pattern classification technique [18], is proposed. Since the vibration of the induction motor is the root cause of motor faults [19], the vibration signal can be analyzed to indicate the state of the motor. The characteristics of vibration signals from the motor in a normal condition are different from those of in a faulty condition. The most common fault detection techniques based on vibration signal focus on the vibration signal's frequencies and magnitude, which are dealing with one dimension domain [7] to [16], [20] to [21]. In this paper, however, other features of the vibration signals that are local features by translating the vibration signal into an image (two dimensions), are explored. The local features from the image are extracted using the SIFT algorithm. The SIFT features, then, are used for the pattern classification process.
The rest of the paper is organized as follows: In Section 1, a short explanation of SIFT algorithm is briefly introduced. Section 2 covers the vibration signal to image translation, feature extraction, framework for pattern classification, and discussion of SIFT feature advantages. The experimental setup and vibration signal database for the fault detection and diagnosis systems are discussed in Section 3. Results and discussions are provided in Section 4, and conclusions in Section 5.

\section{SCALE INVARIANT FEATURE TRANSFORM (SIFT)}

The SIFT algorithm consists of four main filtering stages, which are: scale-space extreme detection, keypoint localization, orientation assignment and keypoint descriptor. In this section, the algorithm containing these four steps is briefly introduced. The details of the SIFT algorithm can be found in [22].

\subsection{Scale-Space Extrema Detection}

The scale space called $L(x, y, \sigma)$ is defined by the following function:

$$
L(x, y, \sigma)=G(x, y, \sigma) * I(x, y),
$$

where, '*' notation is the convolution operator, $G(x, y, \sigma)$ is a variable-scale Gaussian kernel and $I(x, y)$ is the intensity of the pixel, which its coordinates are $\mathrm{x}$ and $\mathrm{y}$. The SIFT is one such technique which locates scale-space extrema from Gaussian image differences called $D(x, y, \sigma)$ given by:

$$
D(x, y, \sigma)=L(x, y, k \sigma)-L(x, y, \sigma),
$$

where $k=1,2,3, \ldots$ is used to present the different scale space. To detect the local maxima or minima of $D(x, y, \sigma)$, each point is compared with its eight neighbors on the same scale, and its nine neighbors on the up and down scale. If this value is larger than all 26 neighbors it is a maxima, if it is smaller then it is minima.

\subsection{Keypoint Localization}

The location of extrema, $z$, is given by: 


$$
z=\frac{-\partial^{2} D^{-1}}{\partial x^{2}} \frac{\partial D}{\partial x} .
$$

If the function value at $z$ is below a threshold value then this point is discarded. This removes extrema that has a low contrast. Edge extrema that have large principle curvatures but small curvatures in the perpendicular direction are eliminated. Using $2 \times 2$ Hessian matrix $H$ computed at the location and scale of the keypoint, principle curvatures which are proportional to eigenvalue of $H$ can be computed.

$$
H=\left[\begin{array}{ll}
D_{x x} & D_{x y} \\
D_{y x} & D_{y y}
\end{array}\right] .
$$

The elimination criteria can be constructed as follows:

$$
\frac{\operatorname{Tr}(H)^{2}}{\operatorname{Det}(H)}<\frac{(r+1)^{2}}{r}, \quad r=\frac{\alpha}{\beta},
$$

where $\sigma$ is Eigenvalue with larger magnitude, and $\beta$ is Eigenvalue with smaller magnitude. If this inequality is true, the keypoint is rejected.

\subsection{Orientation Assignment}

This stage aims to assign a consistent orientation to the keypoints based on local image properties. The keypoint descriptor is represented relative to this orientation because it is invariant to rotational movements of the keypoints. The approach taken to find an orientation has five steps described below:

Step 1: Use the keypoint scale to select the Gaussian smoothed image $L$. Compute gradient magnitude, $m(x, y)$ and orientation, $\theta(x, y)$ by two following Eqs.:

$$
\begin{gathered}
m(x, y)=\sqrt{\begin{array}{l}
(L(x+1, y)-L(x-1, y))^{2}+ \\
(L(x, y+1)-L(x, y-1))^{2}
\end{array}} \\
\theta(x, y)=\tan ^{-1}\left(L(x, y+1)-\frac{L(x, y-1)}{L(x+1, y)-L(x-1, y)}\right) .
\end{gathered}
$$

Step 2: Form an orientation histogram from gradient orientations of sample points. histogram.

Step 3: Locate the highest peak in the

Step 4: Use this peak and any other local peak within $80 \%$ of the height of this peak to create a keypoint with that orientation. Some points will be assigned multiple orientations.

Step 5: Fit a parabola to the three histogram values closest to each peak to interpolate the peaks position.

\subsection{Keypoint Descriptor}

The local gradient data, used above, is also used to create keypoint descriptors. The gradient information is rotated to line up with the orientation of the keypoint and then weighted by a Gaussian kernel with a variance of keypoint scale multiplied by 1.5 . This data is then used to create a set of histograms over a window centered on the keypoint. Keypoint descriptors typically use a set of 16 histograms, which are aligned in a $4 \times 4$ grid, each with eight orientation bins, one for each of the main compass directions and one for each of the mid-points of these directions. These results in a feature vector contain 128 elements.

\section{METHODOLOGY}

\subsection{Vibration Signal to Image Translation}

The proposed approach deals with extracting the features of a vibration signal in twodimension domain. By translating the vibration signal into an image, the local features are extracted using the SIFT algorithm. To translate the vibration signal into an image, the amplitude of each sample of vibration signal is first normalized ranging from 0 to 255 , which is the significant pixel intensity range for a gray image. The intuitive explanation for the translation is indicated in Fig. 2. In this figure, the vibration signal has $M$ multiplying by $N$ samples where the $M \times N$ term is the size of the image ( $M$ and $N$ values are the row and column of the image, respectively). The $M$ and $N$ values are dependent on the length of the vibration signal. However, the computational complexity of the proposed approach will be directly proportional to those values. Therefore, if the complexity is the matter, $M$ and $N$ values should be chosen as small as possible but they should be large enough in order to retain the most significant features from the origin. A recommendation for $M$ and $N$ values is: $M=128 ; 256$ or 512 and $N=128 ; 256$ or 512 . 
The normalized amplitude of each sample point of the vibration signal becomes the intensity of the corresponding pixel in the image. The mapping from normalized amplitude to an equivalent pixel is clearly presented in Fig. 2. The coordinate of the corresponding pixel for the $i^{\text {th }}$ sample in the vibration signal is $\operatorname{pixel}(j, k)$ where $j=$ floor $(i / N)$ and $k=$ modulo $(i / N)$.

An example of a vibration signal from a normal motor translated to a gray image is given in Fig. 3. In this example, the vibration signal has 16384 samples (equivalent to $2.048 \mathrm{~s}$ ) with the sampling rate of $8000 \mathrm{~Hz}$. The translated image size is $128 \times 128$ (i.e. 16384 pixels).

\subsection{Local Feature Extraction and Texton Dictionary}

The SIFT algorithm is used to extract a number of local features from a gray image. Each local feature is a 128-dimension vector, which contain information of location of vector, weight and orientation of each dimension. The details of the SIFT algorithm to extract local feature can be found in [22].

In the proposed fault detection and diagnosis for induction, it has been assumed that all kinds of faulty vibration signals of the induction are known and available in the database (faulty categories). To determine the status of the motor, the testing vibration signal of the motor should be classified into the equivalent faulty category. For classifying, a texton dictionary for each faulty category is used. Each texton dictionary contains the most significant features for that faulty category that are distinctive among the faulty categories. The features from the testing signal will be compared with features in texton dictionaries for classifying based on a pattern classification framework, which will be detailed in Section 2.3.

In the fault detection process, there are two important steps that are feature extraction and texton dictionary creation based on the

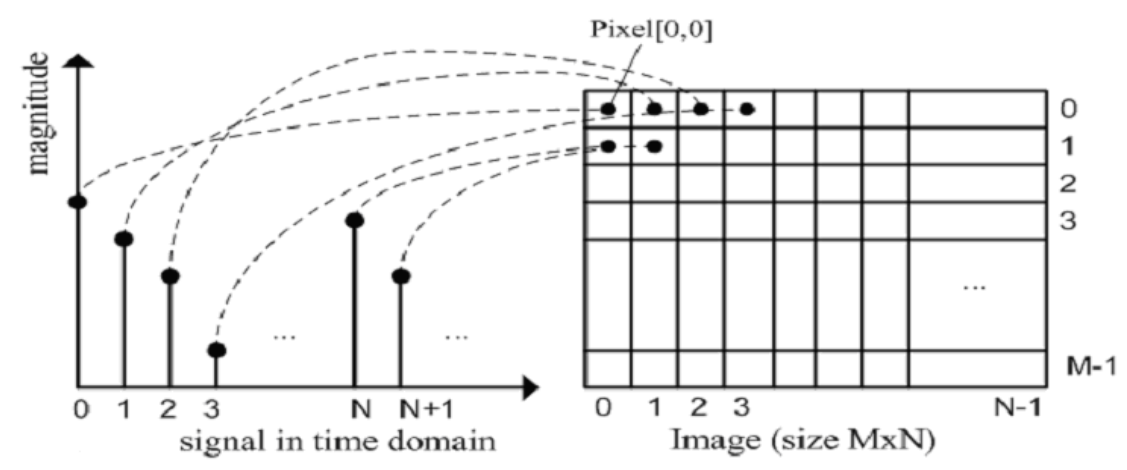

Fig. 2. Vibration signal to image translation scheme
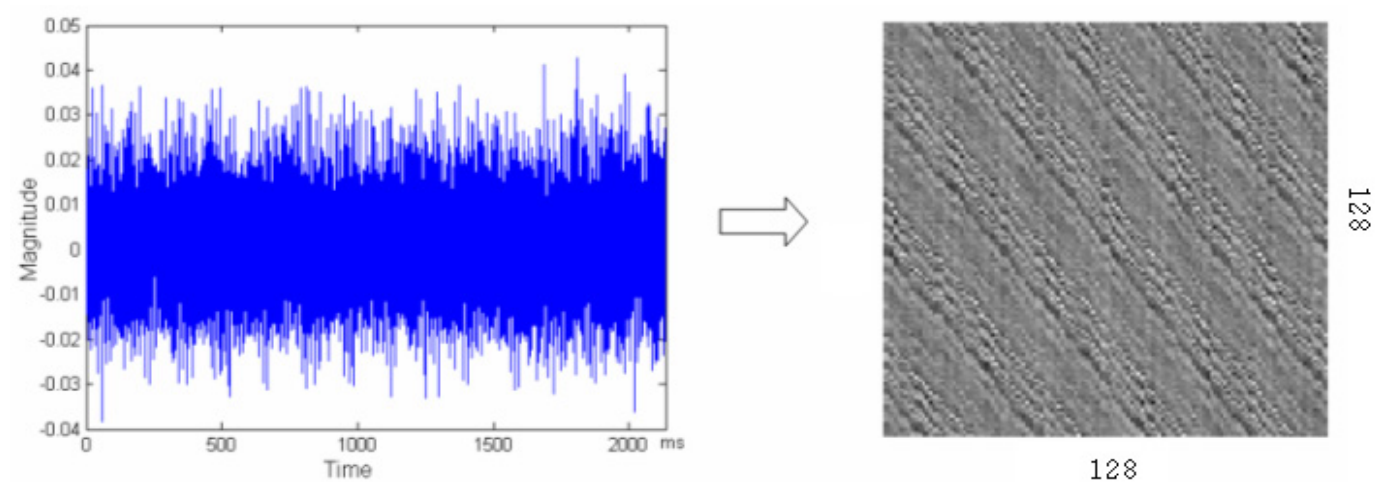

Fig. 3. An example of a vibration signal translated into the $128 \times 128$ gray image $(M=128$ and $N=128)$ 


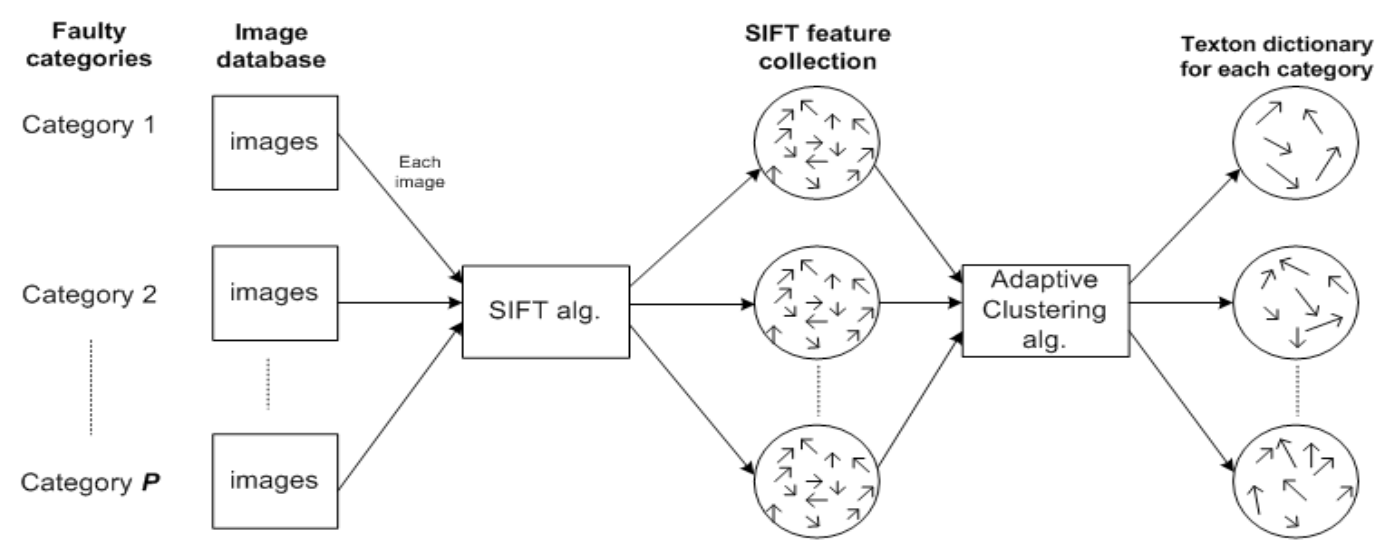

Fig. 4. The model for creating the faulty database

images translated from the vibration signals. As mentioned above, for the feature extraction, the SIFT algorithm is applied for images to generate the 128-dimension feature vectors. The feature vectors are considered as the features of interest. For the texton dictionary creation, a number of images translated from vibration signals for each faulty category are used to create the texton dictionary for that category. The adaptive shift clustering algorithm, which is presented in [23] to [25] is utilized to generate $\boldsymbol{C}$ centroid feature vectors (referred to textons); therefore, the $\boldsymbol{C}$ centroid feature vectors are representative of the category. This collection of $\boldsymbol{C}$ centroid feature vectors is; therefore, called "texton dictionary" for the faulty category. The value $\boldsymbol{C}$ for each category is proportionate to the number of vector feature generated (e.g. each subset of 100 random feature vectors in one category is clustered into 10 textons). Hence, value $\boldsymbol{C}$ for each category is different. The texton dictionary creation procedure is illustrated in Fig. 4 (assuming that there are $\boldsymbol{P}$ faulty categories considered).

\subsection{Pattern Classification Framework in Fault Diagnosis}

In the fault diagnosis process, the feature vectors extracted from the SIFT algorithm as the faulty symptoms are used. Using a pattern classification technique, the current vibration signal should be classified to the corresponding faulty category through a pattern classification framework. The pattern classification framework utilizing the feature vectors and the texton dictionary is proposed as follows:

Step 1: Translate the vibration signal to the image.

Step 2: Apply the SIFT algorithm for this image to generate the 128-dimension feature vectors.

Step 3: Calculate the Euclidean distance between each feature vector and each centroid feature vector in the texton dictionary for each faulty category. With two 128-dimension vectors $V_{1}$ and $V_{2}$ with the coordinates $\left\{V_{1}^{1}, V_{1}^{2}, \ldots, V_{1}^{128}\right\}$ and $\left\{V_{2}^{1}, V_{2}^{2}, \ldots, V_{2}^{128}\right\}$, respectively, the Euclidean distance can be calculated as follows:

$$
D_{12}=\sqrt{\sum_{i=1}^{128}\left(V_{1}^{i}-V_{2}^{i}\right)^{2}} .
$$

Step 4: Find the category containing the centroid feature vector that makes the distance minimal. The centroid feature vector is called the "match" vector.

Step 5: Apply for entire feature vectors of the vibration signal; therefore, the histogram for the "match" vector is created for each faulty category.

Step 6: Classify the vibration signal to the equivalent faulty category based on the created histogram. The vibration signal belongs to the category with the highest number of "match" vector.

The simulation of the detail algorithm is clearly presented in Fig. 5. 


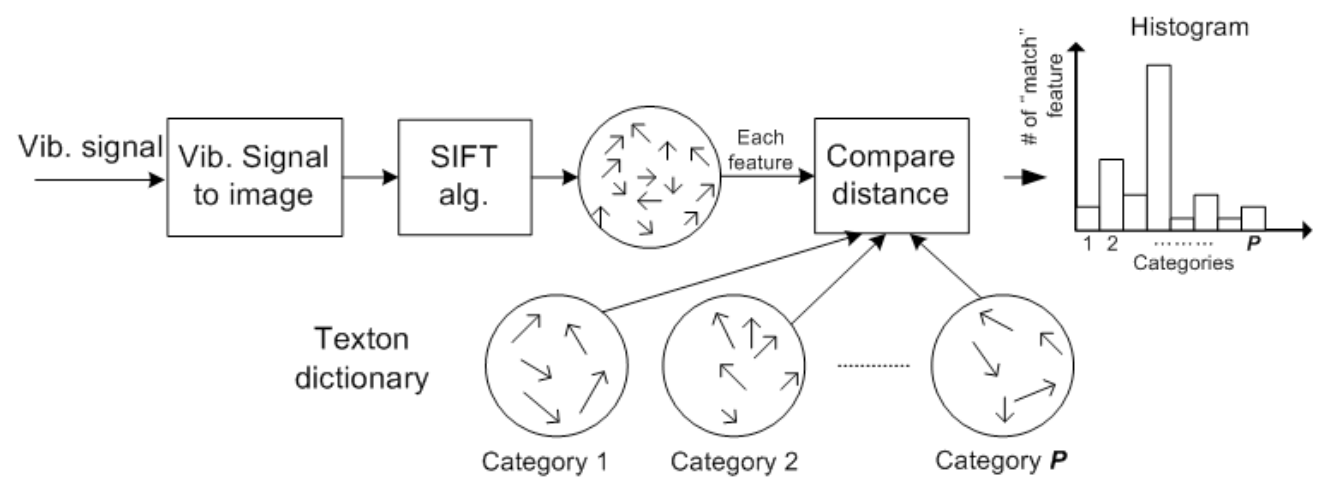

Fig. 5. Framework for classification by model

\subsection{Advantages of SIFT Features}

As mentioned in [22], the SIFT features have some strong characteristics comparing to other local features such as: invariant to image scaling and rotation, partially invariant to change in illumination and 3D camera viewpoint, and highly distinctive.

Machine operating in industrial plants work in noisy environments, and as a result, the useless noise added in the recorded signals is unpreventable. As such, it may be an obstacle for analyzing the vibration signals. However, when the vibration signals are translated into images, the added noise is considered as the illumination of the light to the image. Hence, the effect of noise to the signal is removed when using SIFT features. When using pattern classification technique to classify signals, the highly distinctive characteristic of SIFT feature provides an efficient classification with high accuracy. Those advantages of the SIFT algorithm motivated us exploiting its output features for induction motor fault detection using the classification technique.

\section{EXPERIMENT SETUP AND TRAINING AND TESTING DATABASE}

\subsection{Experiment Setup}

The experiment was setup under a selfdesigned test rig. The experiment consists of motor, pulleys, belt, shaft and fan with changeable blade pitch angle as shown in Fig. 6 (Yang et al, 2006). In the experiment, six $0.5 \mathrm{~kW}, 60 \mathrm{~Hz}$, 4-pole induction motors are used to create the data needed under full load conditions. One of the motors operates under normal as a benchmark for comparison with faulty motors. The others are faulty motors: bowed rotor, broken rotor bar, bearing outer race fault, rotor unbalance, adjustable eccentricity motor (misalignment), and phase unbalance as shown in Fig. 7. Therefore, there are eight kinds of vibration signal categories called "faulty categories"; they are: angular misalignment, bowed rotor shaft, broken rotor bar, faulty bearing (out race), rotor unbalance, normal motor, parallel misalignment and phase unbalance.

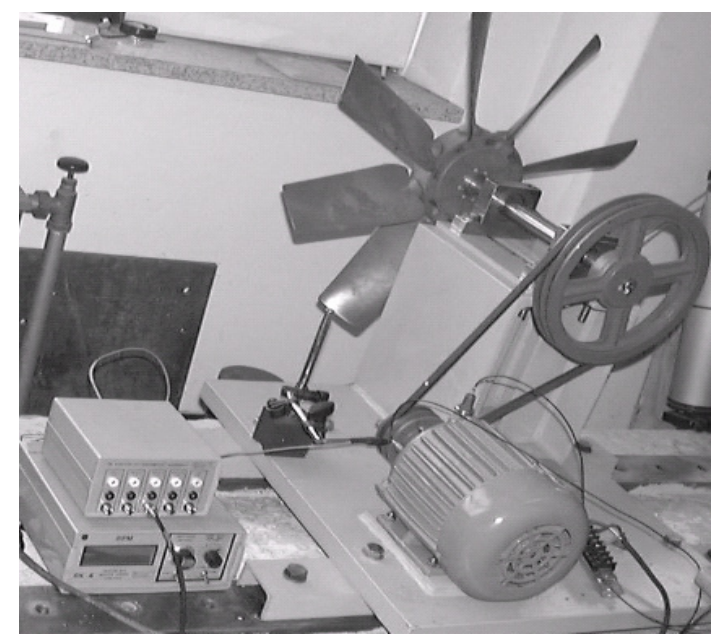

Fig. 6. Experiment setup

The conditions of faulty induction faulty motors are described in Table 1. The motor's load can be changed by adjusting the blade pitch angle or the number of blades. An accelerometer was used to measure the vibration signals of vertical 


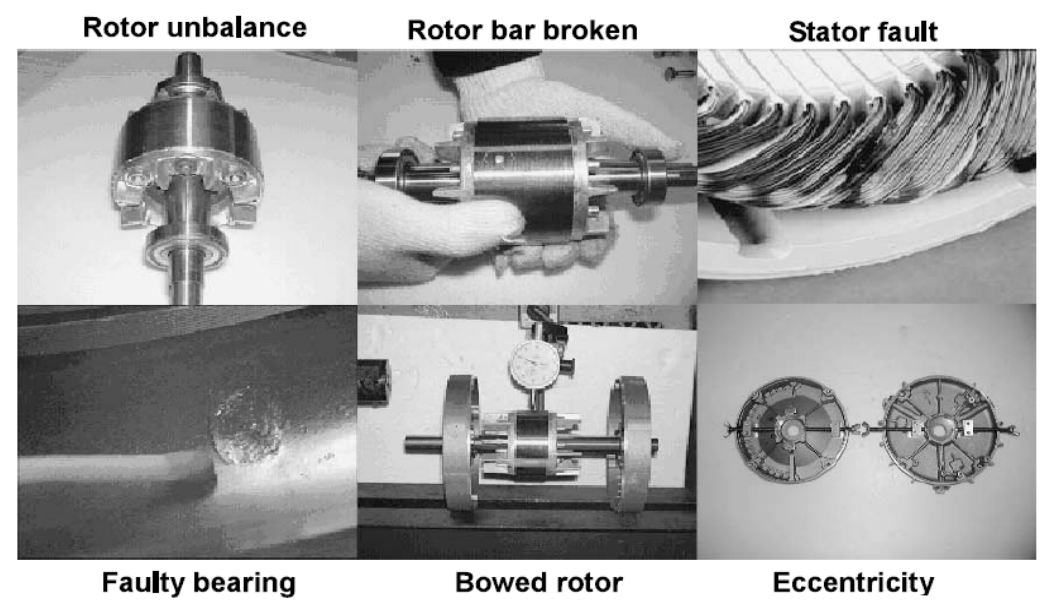

Fig. 7. Faults on induction motor

Table 1. Description of faulty induction motor

\begin{tabular}{|l|l|l|}
\hline \multicolumn{1}{|c|}{ Fault condition } & \multicolumn{1}{c|}{ Fault description } & \multicolumn{1}{c|}{ Others } \\
\hline Broken rotor bar & Number of broken bar: 12 & Total number of 34 bar \\
\hline Bowed rotor & Maximum bowed shaft deflection: $0.075 \mathrm{~mm}$ & Air-gap: $0.25 \mathrm{~mm}$ \\
\hline Faulty bearing & A spalling on outer raceway & $\# 6203$ \\
\hline Rotor unbalance & Unbalance mass on the rotor $(8.4 \mathrm{~g})$ & \\
\hline Eccentricity & Parallel and angular misalignments & Adjusting the bearing pedestal \\
\hline Phase unbalance & Add resistance on one phase & $8.4 \%$ \\
\hline
\end{tabular}

direction. In this paper, the vibration signals are used to evaluate the proposed fault detection and diagnosis algorithm. The sampling frequency of the experiment is $8 \mathrm{Khz}$; therefore, the maximum frequency obtained is $4 \mathrm{Khz}$. The vibration signal contains low frequency components; hence, vibration signal with $8000 \mathrm{~Hz}$ sampling rate is fully reasonable to consider. Each vibration signal consists of 16384 data points.

\subsection{Training and Testing Database}

For an evaluation of the proposed fault detection and diagnosis, two vibration signal databases are required: training and testing databases. The training database is used for creating the texton dictionary, while the testing database is for testing the efficiency of the proposed approach based on the accuracy of the classification. In this experiment, eight vibration signals were collected from each faulty category from which two random signals for training database and six the other for testing database. So the testing database consists of 48 vibration signals with six signals for each category, while the training database has 16 signals with 2 signals for each category. An example of waveforms of eight vibration signals from eight faulty categories and their translated images is illustrated in Fig. 8.

As mentioned above, the training database to construct the texton dictionary is used to represent the main characteristics of each category by a collection of centroid feature vectors after using the clustering algorithm. A trained vibration signal from the testing database is analyzed to classify in the corresponding faulty category using our proposed pattern classification framework. Each faulty category name is denoted as follows. Am: Angular misalignment, Br: bowed rotor shaft, $\mathrm{Brb}$ : broken rotor bar, $\mathrm{Fb}$ : faulty bearing, Mu: rotor unbalance, No: normal motor, Pm: parallel misalignment and $\mathrm{Pu}$ : phase unbalance. 

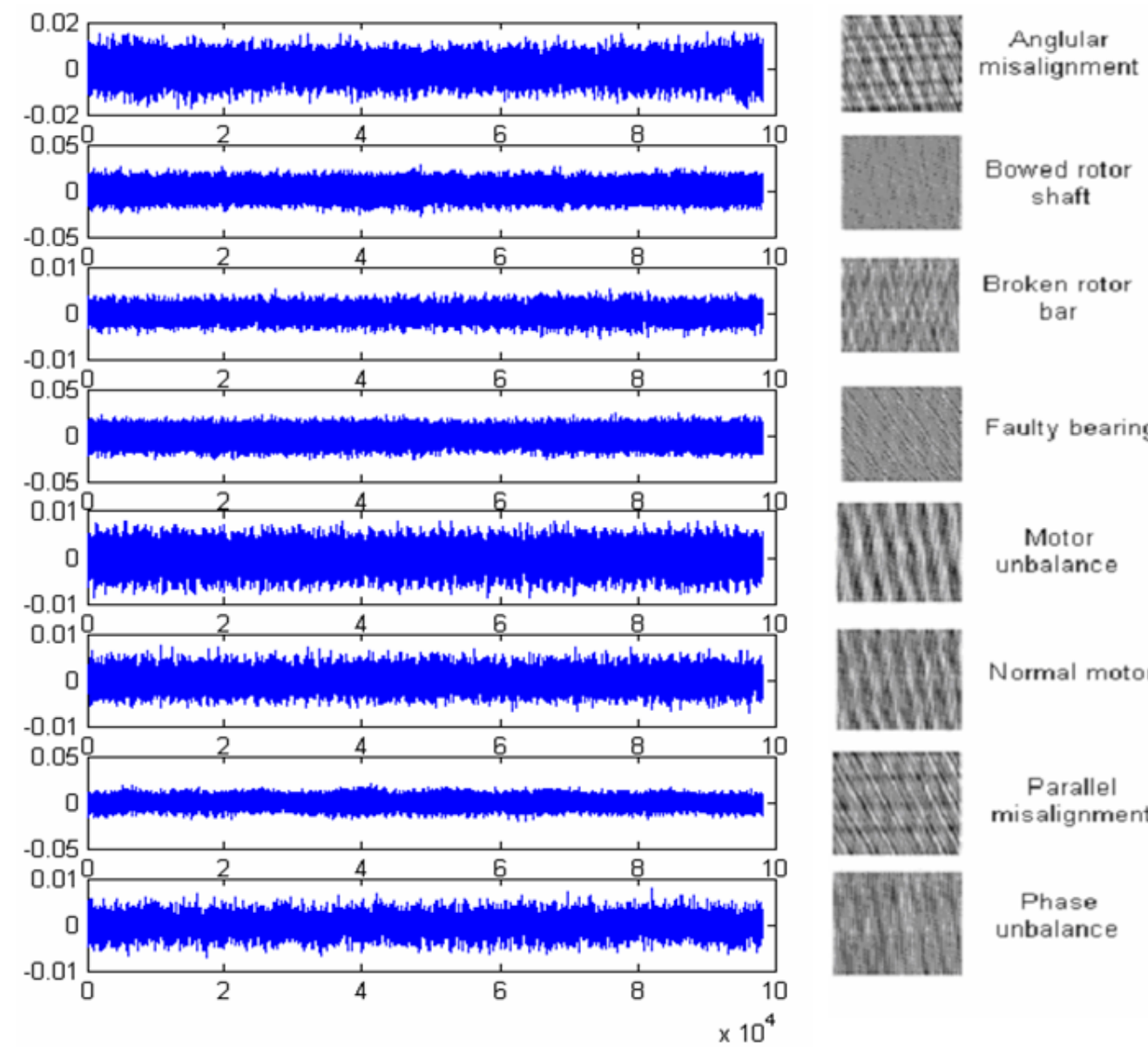

Broken rotor

bar

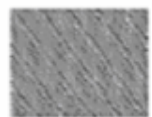

Faulty bearing

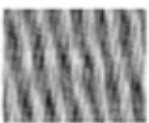

Motor unbalance

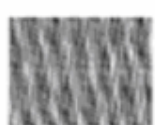

Normal motor

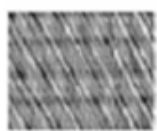

Parallel

misalignment

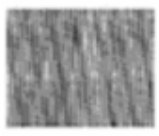

Phase

unbalance

Fig. 8. Waveforms of eight vibration signal samples from eight faulty categories and their equivalent translating images

\section{RESULTS AND DISCUSSIONS}

In the experiment, a classification for faulty vibration signals from machines is implemented. In our test, 10 trials were carried out. For each trial, two randomly selected signals are for training database and six others for testing, therefore, the testing database consisting of 48 vibration signals of eight faulty signal categories (Am, Br, Brb, $\mathrm{Fb}, \mathrm{Mu}, \mathrm{No}, \mathrm{Pm}$, and $\mathrm{Pu}$ ) with six signals for each, are used for training. Each vibration signal in the testing database is used to extract the SIFT features. The classification framework proposed in section 2.3 is applied with the features and texton dictionary in order to classify the vibration signal to the corresponding faulty category.
Fig. 9 depicts an example of a classification for a vibration signal in the testing database, which is supposed to classify in the broken rotor bar category. In this example, 610 feature vectors are created after using the SIFT algorithm. The figure indicates that in the 610 feature vectors, there are 30 "match" vectors for Am, 4 for Br, 207 for Brb, 20 for $\mathrm{Fb}, 68$ for $\mathrm{Mu}, 123$ for No, 33 for $\mathrm{Pm}$, and 125 for $\mathrm{Pu}$ categories discovered in the texton dictionaries, respectively. The histogram indicates that the trained signal is classified in the broken rotor bar category because of the highest "match" vectors number.

Table 2 provides a local feature distribution of each signal for each faulty vibration signal classification when a representative of each category is trained. The percentage of local 


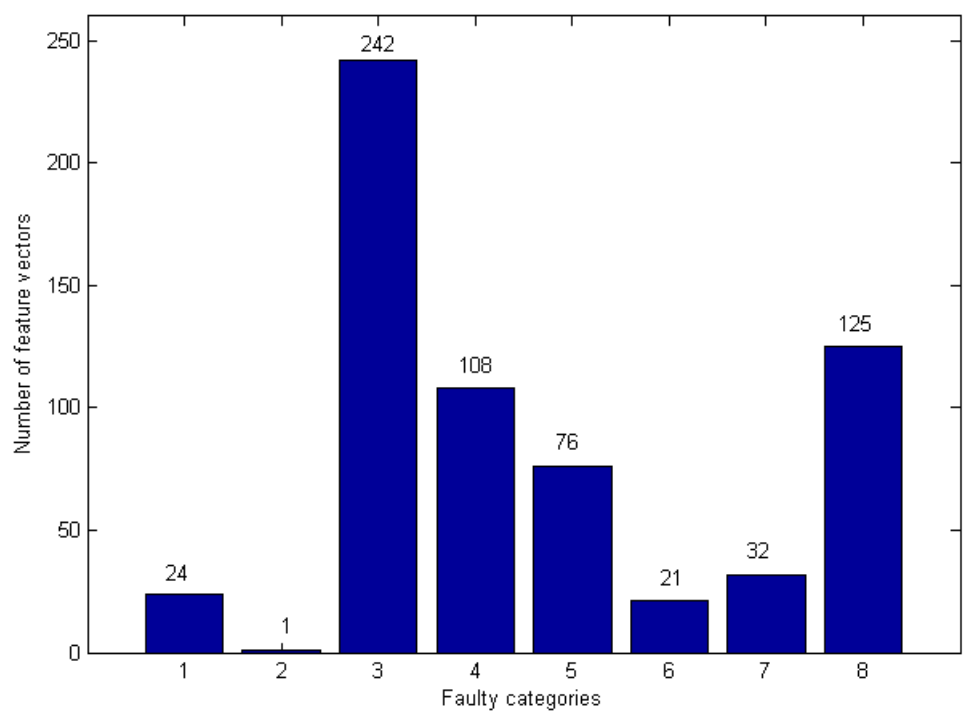

Fig. 9. Histogram for feature vectors of a vibration signal from the testing database; on the Xaxis, 1: Am, 2: $\mathrm{Br}, 3: \mathrm{Brb}, 4: \mathrm{Fb}, 5: \mathrm{Mu}$, 6: No, 7: Pm and 8: Pu faulty categories

Table 2. Percentage of local feature distribution

\begin{tabular}{|l|c|c|c|c|c|c|c|c|}
\hline \multirow{2}{*}{$\begin{array}{c}\text { Signal (Belonged } \\
\text { category) }\end{array}$} & \multicolumn{9}{|c|}{ Faulty categories } \\
\cline { 2 - 9 } & $\mathrm{Am}$ & $\mathrm{Br}$ & $\mathrm{Brb}$ & $\mathrm{Fb}$ & $\mathrm{Mu}$ & Nor & $\mathrm{Pm}$ & $\mathrm{Pu}$ \\
\hline $1(\mathrm{Am})$ & 44.66 & 2.16 & 6.47 & 4.75 & 8.20 & 5.39 & 16.40 & 11.97 \\
\hline $2(\mathrm{Br})$ & 0.78 & 91.47 & 0.00 & 6.98 & 0.00 & 0.00 & 0.77 & 0.00 \\
\hline $3(\mathrm{Brb})$ & 4.34 & 0.16 & 37.21 & 2.79 & 13.80 & 14.11 & 5.89 & 21.70 \\
\hline $4(\mathrm{Fb})$ & 0.81 & 4.40 & 2.77 & 75.37 & 8.32 & 3.26 & 2.60 & 2.45 \\
\hline $5(\mathrm{Mu})$ & 2.37 & 0.00 & 10.53 & 4.01 & 42.73 & 14.54 & 3.41 & 22.40 \\
\hline $6(\mathrm{Nor})$ & 4.67 & 0.81 & 13.69 & 7.24 & 18.04 & 34.46 & 4.51 & 16.59 \\
\hline $7(\mathrm{Pm})$ & 27.12 & 1.84 & 3.80 & 6.75 & 4.05 & 3.19 & 50.80 & 2.45 \\
\hline $8(\mathrm{Pu})$ & 3.83 & 0.12 & 15.21 & 1.97 & 20.79 & 11.61 & 1.74 & 44.71 \\
\hline
\end{tabular}

feature distribution represents the probability of the number of features of training vibration signal falling into one faulty category. The highest percentage number indicates the corresponding faulty categories that the vibration signal belongs to (the highest number in a row in Table 2). In Table 2, eight arbitrary vibration signals from eight faulty categories from testing database are selected for training and they are all correctly recognized.

Table 3 shows the final classification results for 10 trials. The average of total classification accuracy after 10 trials reaches $97.9 \%$, which is considerably high. There are four among ten trials even giving $100 \%$ of accuracy. In the worse trial (trial \#7), the classification accuracy of
$93.7 \%$ is even sufficient enough to be accepted in fault detection and diagnosis with classification technique.

To compare with other approaches in our previous reseach, we used two approaches introduced in [26], which are one-dimension domain and are wavelet-variance based and wavelet-crosscorelation based approaches. For experimental data in this paper, the result is shown in Table 4. The higher classification accuracy of the proposed approach clearly shows a big advantange of exploying two-dimension domain features over that of one-dimension domain.

Another comparison of these three approaches was also caried out. The experimental data were taken from [26]. The data are in 
Table 3. Classification accuracy of proposed approach

\begin{tabular}{|c|c|c|c|c|c|c|c|c|c|}
\hline \multirow{3}{*}{ Trials \# } & \multicolumn{8}{|c|}{ Faulty category name (number of testing signals) } & \multirow{3}{*}{$\begin{array}{c}\text { Total } \\
\text { classification } \\
\text { accuracy }[\%]\end{array}$} \\
\hline & $\operatorname{Am}(6)$ & $\operatorname{Br}(6)$ & $\operatorname{Brb}(6)$ & $\mathrm{Fb}(6)$ & $\mathrm{Mu}(6)$ & Nor(6) & $\operatorname{Pm}(6)$ & $\mathrm{Pu}(6)$ & \\
\hline & \multicolumn{8}{|c|}{ Number of sucessful testing signals classified to corresponding categories } & \\
\hline 1 & 6 & 6 & 6 & 6 & 6 & 5 & 6 & 6 & 97.9 \\
\hline 2 & 6 & 6 & 6 & 6 & 6 & 6 & 6 & 6 & 100 \\
\hline 3 & 6 & 6 & 6 & 6 & 6 & 4 & 6 & 6 & 95.8 \\
\hline 4 & 6 & 6 & 6 & 6 & 6 & 6 & 6 & 6 & 100 \\
\hline 5 & 6 & 6 & 6 & 6 & 6 & 5 & 6 & 6 & 97.9 \\
\hline 6 & 6 & 6 & 6 & 6 & 6 & 6 & 6 & 6 & 100 \\
\hline 7 & 6 & 6 & 5 & 6 & 6 & 4 & 6 & 6 & 93.7 \\
\hline 8 & 5 & 6 & 6 & 6 & 6 & 5 & 6 & 6 & 95.8 \\
\hline 9 & 6 & 6 & 6 & 6 & 6 & 6 & 6 & 6 & 100 \\
\hline 10 & 6 & 6 & 6 & 6 & 6 & 5 & 6 & 6 & 97.9 \\
\hline \multicolumn{9}{|c|}{ Total average of classification accuracy for 10 trials } & 97.9 \\
\hline
\end{tabular}

Table 4. Comparison of proposed approach and two approaches proposed in [26] in term of classification accuracy

\begin{tabular}{|c|c|c|c|}
\hline & \multicolumn{3}{|c|}{ Approaches } \\
\cline { 2 - 4 } & Proposed approach & $\begin{array}{c}\text { Wavelet-Variance } \\
\text { based approach }\end{array}$ & $\begin{array}{c}\text { Wavelet-Crosscorrelation } \\
\text { based approach }\end{array}$ \\
\hline $\begin{array}{c}\text { Average classification } \\
\text { accuracy for 10 trials [\%] }\end{array}$ & 97.9 & 89.3 & 78.6 \\
\hline
\end{tabular}

Table 5. Comparison of proposed approach and two approaches proposed in [26] in term of classification accuracy using experiment data in [26]

\begin{tabular}{|c|c|c|c|}
\hline & \multicolumn{3}{|c|}{ Approaches } \\
\cline { 2 - 4 } & Proposed approach & $\begin{array}{c}\text { Wavelet-Variance based } \\
\text { approach }\end{array}$ & $\begin{array}{c}\text { Wavelet-Crosscorrelation } \\
\text { based approach }\end{array}$ \\
\hline $\begin{array}{c}\text { Average classification } \\
\text { accuracy for 10 trials [\%] }\end{array}$ & 98.1 & 95.6 & 78.7 \\
\hline
\end{tabular}

microphone format (sound data taken by microphone). In these data, five sound data types from five different faulty categories of induction motors are: fault bearing, loose bearing, unbalance bearing, misalignment bearing and normal bearing. The details of experiment setup and data can be found in [26]. Three approaches are applied with this data. Again, 10 trials of testing were applied. Then, final classification accuracy is shown in Table 5. The avarage classification accuracy from 10 trials for proposed approach gains $98.1 \%$ compared to 95.6 and $78.7 \%$ for two other ones, respectively. Once again, with this different experiment, the proposed approach provides a better performance.

\section{CONCLUSIONS}

The results of high classification accuracies achieved by the proposed approach clearly demonstrates the potential of exploiting the features from two-dimension domain data of vibration signal by considering the SIFT algorithm for fault detection and diagnosis system. Different from the previous approaches for a fault detection and diagnosis using features of vibration signal in one-dimension domain, we propose a vibration signal to image translation technique and a classification framework that are applied in a novel fault detection and diagnosis system for induction motors. For a real application, 
knowledge about the induction motor faults should be fulfilled; therefore, the most common faults needs to be examined and can be represented in the texton dictionary. The results indicate that some features of the testing signal belong to other faulty categories; however, most of features are classified to the corresponding ones so the testing signal is still successfully classified into the correct faulty category. The high classification accuracy in the final results indicates that our proposed fault detection and diagnosis using signal model-based exploiting vibration signal in two-dimension domain is guaranteed.

\section{REFERENCES}

[1] Isermann, R. (1994). Integration of fault detection and diagnosis methods. IFAC Symposium on fault detection, Supervision and Safety for Technical Processes, Espoo, p. 587-609.

[2] Isermann, R. (1997). Supervision, fault detection and diagnosis methods - an introduction. Control Engineering Practice - CEP, vol. 5, no. 5, p. 639-652.

[3] Isermann, R. (2006). Fault diagnosis system: An introduction from fault detection to fault tolerance. Springer, Berlin.

[4] Kimmich, F., Schwarte, A., Isermann, R. (2005). Fault detection for modern diesel engines using signal-and process modelbased methods. Control Engineering Practice, vol. 13, p. 189-203.

[5] Kimmich, F., Schwarte, A., Isermann, R. (2001). Model based fault detection for diesel engines. Aachen Colloquium, Aachen.

[6] Isermann, R. (1984). Process fault detection on modeling and estimation methods - a survey. Automatica, p. 387-404.

[7] Virtic, M., Abersek, B., Zuperl, U. (2008). Using of acoustic models in mechanical diagnostics. Strojniški vestnik - Journal of Mechanical Engineering, vol. 54, no. 12, p. 874-882

[8] Žumer, J., Biček, A., Boltežar, M. (2008). Characterization of the vibrations and structural noise of a suction unit's cover. Strojniški vestnik - Journal of Mechanical Engineering, vol. 54, no. 12, p. 883-891.
[9] Furlan, M., Rebec, R., Cernigoj A., Celic, D., Cermelj, P., Boltezar, M. (2006). Vibro-acoustic modelling of an alternator. Strojniški vestnik - Journal of Mechanical Engineering, vol. 52, no. 2, p. 112-125.

[10]Boyle, C. (1994). Online current monitoring to detect misalignment and dynamic eccentricity in three-phase induction motor drives. 29th universities power engineering conference 1, p. 5-8.

[11] McInerny, S.A., Dai, Y. (2003). Basic vibration signal processing for bearing fault detection. IEEE Transaction on Education, vol. 46, no. 1, p. 149-156.

[12]Kahaei, M., Torbatian, M., Poshtan, J. (2007). Bearing-fault detection using the Meyer-wavelet-packets algorithm. Strojniški vestnik - Journal of Mechanical Engineering, vol. 53, no. 3, p. 186-192.

[13] Arenas, J.P. (2005). Enhancing the vibration signal from rolling contact bearing using an adaptive closed-loop feedback control for wavelet de-noising. Strojniški vestnik Journal of Mechanical Engineering, vol. 51, no. 4, p. 184-192.

[14]Belsak, A., Flasker, J. (2008). Vibration analysis to determine the condition of gear units. Strojniški vestnik - Journal of Mechanical Engineering, vol. 54, no. 1, p. 11-24.

[15] Yang, B.S., Han, T., Yin, Z.T. (2006). Fault diagnosis system of induction motor using feature extraction, feature selection and classification algorithm. JSME International Journal, vol. 49, no. 3, p. 734-741.

[16] Goncalves, V.D., Almeida, L.F., Mathias, M.H. (2010). Wear Particle Classifier System Based on an Artificial Neural Network. Strojniški vestnik - Journal of Mechanical Engineering, vol. 50, no. 4, p. 284-288.

[17]Patan, K. (2008). Artificial neural networks for the modeling and fault diagnosis of technical processes. Springer Verlag, Berlin.

[18]Earl, G., Richard, J., Steve, J. (1996). Pattern recognition and image analysis. Prentice Hall, New York.

[19] Micheal, N., Denis, K. (2003). Fundamentals of noise and vibration analysis for engineers. Cambridge University Press, Cambridge. 
[20]Benbouzid, M.E.H. (2000). A review of induction motors signature analysis as a medium for faults detection. IEEE Transactions on Power Electronics, vol. 14, no. 5, p. 984-993.

[21] Ocak, H., Loparo, K.A. (2004). Estimation of the running speed and bearing defect frequencies of an induction motor from vibration data. Mechanical Systems and Signal Processing, vol. 18, no. 3, p. 515-533.

[22] Lowe, D. (2004). Distinctive image features from scale-invariant keypoints. International Journal of Computer Vision, vol. 60, no. 2, p. 91-110.

[23]Krystian, M., Cordelia, S. (2005). A performance evaluation of local descriptors. IEEE Trans. Pattern Analysis and Machine Intelligence, vol. 27, no. 10, p. 1615-1629.
[24]Leung, T., Malik, J. (2001). Representing and recognizing the visual appearance of materials using three-dimensional textons. International Journal of Computer Vision, vol. 43 , no. 1, p. 29-44.

[25]Georgescu, B., Shimshoni. I., Meer, P. (2003). Mean shift based clustering in high dimensions: a texture classification example. $9^{\text {th }}$ IEEE International Conference on Computer Vision 1, p. 456-463.

[26] Do V.T., Cho S.J., Chong U.P. (2009). Fault detection and diagnosis for induction motors using variance, crosscorrelation and wavelets. Transactions of Korean Society for Noise and Vibration Engineering, vol. 19, no. 7, p. 726735. 